Portanto, os actuaes conhecimentos sobre a leucogenese post fetal, a estatica e a pathologia do sangue, permittem seja conferida ao monocyto a cathegoria de terceira especie de cellula branca, madura, independente e da mesma hierarchia dos leucocytos polymorpho granulosos e dos lymphocytos.

\title{
Clinica Obstetrica
}

\author{
(Notas de aula pratica)
}

Dr. Raul Briquet. (assistente de clinica obstetrica)

As notas, cuja publicação hoje iniciamos, têm por exclusivo objecto orientar os srs. alumnos na technica dos exercicios tocomaticos.

$\mathrm{Na}$ pratica segura das intervenções obstetricas importa, antes de mais, o conhecimento dos phenomenos do trabalho, em especial, do mecanismo do parto.

Este, em essencia, reduz-se a um problema de dynamica. Já em 1753, dizia Levret que "o trabalho de parto é uma opperação natural, verdadeiramente mecanica, susceptivel de uma demonstração geometrica"

Tres são os seus factores: $10^{\circ}$ a potencia (contracção uterina, e, subsidiariamente, a do diaphragma e a dos musculos da parede antero-lateral do abdome); $2 .^{\circ}$ o canal; $3 .^{\circ}$ o movel (féto e annexos).

Só trataremos dos dois ultimos, pois que o exame do $1 .^{\circ}$ melhor se fará na assistencia ao parto, em que será realizado sob o ponto de vista clinico e experimental.

\section{Bacia (factor do trabalho)}

Assim se designou a cavidade circumscripta pela columna sacrocócica atrás, pelo iliacos, adeante e aos lados. Recordava aos antigos, pela conformação, o prato ou bacia de que se serviam os barbeiros.

E' grande ou pequena, conforme assestada acima ou abaixo do estreito superior.

Aos obstetras a grande bacia é de pequeno interesse.

A pequena bacia ou pelve obstetrica é formada por dois segmentos sobrepostos: um, osiseo, revestido de paredes molles - bacia obstetrica propriamente, ou escavação; outro, fibro-muscular, infundibulo perineo-vulvar, bacia molle ou dilatavel, de alguns autores.

\section{Bacia obstetrica ou escavação}

E' limitada, em cima, pelo estreito superior, (que a isola da grande bacia), e, em baixo, pelo inferior. Estrictamente, o seu limite superior é o plano transverso-pubico de Fochier, (borda su- 
perior da symphyse e $2 .^{\text {a }}$ sacra, ou, segundo outros, o plano terminal de Froriep, que passa pelas linhas innominadas ou terminaes.

0 parteiro precisa saber daquelles planos do canal pelvi-genital que possam, pela sua maior proeminencia na cavidade da bacia, restringir-lhes os diametros.

A.cceito este ponto de vista, perde de importancia o eistreito superior anatomico, cujos pontos constituintes são: borda superior da symphyse púbica, borda superior do ramo horizontal do pube, linha innominada, borda anterior da asa do sacro, e a do angulo sacro-vertebral. Elle não corresponde, com effeito, á angustia dimensória separativa da grande e pequena bacia, nem tampouco as suas diversas regiões anatomicas residem em um e mesmo plan.o.

'Clinicamente, o estreito superior é representado 'pela angustia superior de Demelin, a qual toca os pontos mais salientes das symphyses isacro-iliacas e a borda posterior das gotteiras obturadoras.

Tem a fórma de um coração de carta de jogar, nelle predominando os diametros transversos.

o estreito inferior vae do sob-pube, borda inferior dos ramos ischio-pubicos, face interna das tuberosidades ischiaticas, ligamentos sacro-sciaticos á extremidade do cócix.

Neste, o maior diametro é o antera-posterior, de feição que, de cima a baixo, do estreito superior a inferior, os diametros transversos se vão reduzindo, emquanto os antero-posteriores crescem em tamanho.

No dispositivo do E. S., convem pôr em relevo a arcada púbica, delimitada pelo ligamento anqueado e pelos ramos ischio-pubicos. E' triangular e accusa, em regra, 8 cts. de altura por 11 de base.

A pratica preceitúa: quanto mais alta a symphyse mais se recalcará o perineo, e maís frequentes se farão as dilacerações. Quanto mais larga a arcada púbica, tanto mais garantido se achará o perineo.

Parede. A excavação é conformada: adeante, pela symphyse púbica, superficie angular dos pubes e ramos ischio-pubicoś; atrás, pela face anterior do sacro e do cocix, sylmphyses sacro-iliacas e articulações sacro-cócica e medio-cócica; lateralmente, pela $\operatorname{ch}^{-n}$ fradura sciatica, fundo do cótylo, face interna dås tuberosidades ischiaticas e orificios ischio-pubicos.

As paredes lateraes da escavação, (cuja altura é de 9-10 cts.), são ligeiramente inclinadas de cima para baixo e de fóra para dentro. Foram divididas em duas metades, anterior e posterior, por uma linha que passaria pela base das espinhas sciaticais. Essas zonas têm o nome de planos inclinados; seu valor será apontado ao tratarmos do mecanismo do parto.

Estreito médio. A ssuperficie endo-pelvica da escavação offerece, ao nivel das espinihas sciaticas, uma estenose que mereceu, de certos autores, o nome de estreito médio. Formam-n-o: a ponta do sacro, a apophyse transiversa da $5 .^{\mathrm{a}}$ sacra, a borda do pequeno ligamento sacro-sciatico, la espinha sciatica e a linha que se prolonga desta ultima á união do $1 \mid 3$ inferior com o $1 \mid 3$ médio da face posterior da symphyse pubica.

o estreito medio separa a bacia ossea da molle, e, em seu contorno, processam-se as inserções do diaphragma pelvico.

Altura. A altura da pequena bacia é: adeante (symphyse pubica) de $41 / 2$ cts., embora Demelin affirme que a media nunca é superior a $3.8 \mathrm{mms}$; atrás (concavidade sacro-cócica) 14-15 cts.; late- 
ralmente (da linha innominada á borda inferior da tuberosidade ishiatica), $9-10$ cts.

Pela inflexibilidade topographica que possue, a bacia ossea, como o diz Magalhães, é que mais caracteriza a mecanica da parturição.

Segmento perineo-vulvar. Accrescentado ao E. I., e mantendo a continuidade do canal pelvi-genital, encontra-se 0 infundibulo vagino-perineo-vulvar ou, simplesmente, perineo-vulvar.

Afunilado, tem este segmento a sua entrada constituida pelas inserções do elevador coci-perineal, unidadie muscular resultante da fusão do ischio-cócico, atrás, com o elevador do anus, adeante.

Funcciona qual diaphragma ou soalho pelvico; é muscular, convexo em sua face inferior, possuindo, adeante e na linha mediana, uma fenda, por onde transitam a uretra, a vagina e o recto.

Esta brecha é o orificio pubo-pre-anal ou retro-vulvar. Farabeuf e Varnier dão-lhe o nome de pubo-cócico, denominação que, as ver de Devraigne e Descomps, é inadmissivel, visto não existir tal fenda na especie humana, ou siquer nos anthropoides. Com o surto da attitude erecta, do encurtamento e da parada evolutiva do segmento caudal da columna vertebral, escrevem elles, os musculos flexores da cauda se atrophiam e soldam-se atrás do anus, formam o raphe ano-cócico, reforçam o esphincter externo do anus e sustêm as visceras pelvicas.

Ao diaphragma pelvico muscular annexa-se outro - diaphragma accessorio ou uro-genital, áquelle sotoposto, se olharmois a bacia de baixo para cima. E' este novo systema constituido pelos seguintes musculos: esphincter externo da uretra, transverso profundo do perineo, bolbo-cavernoso, constrictor da vagina, ischio-cavernoso. e esphincter externo do anus.

O diaphragma pelvico tem duas faces: anterior ou sob-pubica, e perineal ou posterior. Esta é a mais importante; da articulação sacro-cócica attinge a commissura posterior da vagina, e, de tal modo se mostra extensivel, que póde exceder $15 \mathrm{cts}$. de comprimento.

Por fim, o anel vulvar é a quarta angustia que se depara no trajecto pelvi-genital.

Linha de progressão. Ao percorrer o canal pelvi-genital, o féts descreve uma trajectoria, determinada pela serie de pontos que unem o centro dos successivos planos do canal.

Chamam-lhe eixo da bacia. A impropriedade da expressão resalta. dil-o Demelin, se attentarmos a que, no caso, nada existe que, sob o aspecto geometrico ou mecanico, recorde a recta em torno da qual gira um plano para crear um solido ou uma superficie, ou aquella liniha mathematica, virtual, em derredor da qual se verifica um movimento.

Mais acertado é denominal-a linha de direcção ou de progressão.

Até ás provas experimentiaes e demolidoras de Fabbri, Boissard, Hodge, admittiam os parteiros a curva de Carus (arco de circulo de concavidade anterior, indo do E, S. ao anel vulvar, como representativa do caminho seguido pelo féto em sua passagem através do conducto utero-vaginal.

Boisıard figurava a linha de progressão por duas rectas: uma, limha de insinuação ou de descida: outra, de desprendimento, ambas formando um angulo de grande abertura anterior.

Para Hodge, a linha de direç̧ão é composta de duas rectas unidas por ligeira linha curva. Esta é a liç̧ão de Devraigne e Descomps (pag. 22 do Trat. de Bar, Brindeau, Chambrelet $1 .^{\circ}$ vol 1914). 
Não coincide, entretanto, com o ensinamento de Lee (pg. 166 do seu tratado, de 1913), e cuja exposição julgamos mais consentanea com a.s demonstrações do illustre obstetra de Philadelphia.

Assim se exprime Lee: "A study of the parturient canal will show that it runs straight down until it reaches the narrow pelvic plane or the third parallel of Hodge, then bends forward in a sharp curve whose is the symphyse pulis. If the passage uses the space under the arch of the pubis, the bend in it is quite sharp. If the arch of the pubis is narrow, or if the fetal head is very large, the occiput stems on the ramis pubis, forcing the pelvic floor further back toward the sacrum, making a more obtuse angle in the parturient canal. The axis of the parturient canal is, therefore, not a curve (the curve of Carus), but a straight line with a bend at the pelvic floor and a curve."

Assim posto o assumpto, a concepção de Sellheim e, em regra, a dos allemães, é a mesma do parteiro americano. A linha de progressão é. constituida por duas linhas: a primeira, recta, de descida corresponde ao cylindro superior (borda superior á inferior da symphyse pubica); a segunda, curva, de desprendimento, com a concavidade voltada ao sob-pube.

De um modo geral, a linha de direção traduz os contactos successivos da parte fetal com o arco anterior da bacia. Não se juxtapõe á linha central da escavação, mas passa um pouco adeante. Conforme o maior ou menor volume da cabeça, ella se achará mais ou menos distante da symphyse pubica.

Demelin cuidou com attenção deste ponto.

Theoricamente, a linha de insinuação dista 5 cts. da face posterior da symphyse pubica; quando a parturiente está deitada, é quasí vertical, em seguida inflecte-se, torna-se horizontal, rectificando-se para cima, por fím. Muitas vezes, excentrica, está mais chegada de de uma ou outra parede lateral, aproximando-se, em baixo, do plano mediano antero-posterior da bacia.

A linha de desprendimento occupa este ultimo plano; é curvilinea, de concavidade a principio superior, em seguida, postero-superior.

$\mathrm{Na}$ parturiente, em decubito dorsal, ao iniciar-se a inßinuação, a linha de direcção é obliqua de cima para baixo, e de trás para deante, muito mais proxima da vertical que da horizontal.

'A' medida que se opera a descida, a linha de progressão relaciona-se com a horizontal, em a qual se funde, logo que a região fetal transponha a arcada pubiea. Por fim, o perineo reage e imprime á linha de direcção novo impulso que, de baixo para cima e de trás para deante, irá conduzil-a á vertical.

Topographicamente, o segmento perineo-vulvar representa a linha de desprendimento, e a bacia osisea, a de insinuação, ou de progressão.

O féto, em sua expulsão utero-vaginal, percorre o diaphragma cervico-segmentario, no periodo de dilatação, isto é, a linha de insinuação ou de descida, e o cóci-perineal, na expulsiva, e, portanto, a de desprendimento.

Cumpre conhecer a direcção seguida pelo féto em seu eixo do conducto genital, afim de acompanhar-se o parto e praticar-se, seguindo-a, as necessarias operações extractivas.

Linha central da escavação. $\mathrm{E}^{\prime}$ aquella que passa pelo plano sagital da escáva, a igual distancía da face posterior da symphyse e anterior da columna sacro-cócica.

Revela a direção da pequena bacia, e ha quem the chame eixo da bacia. 
E' sempre unica e immutavel, ao passo que a linha de direç̧ão varia com as circumstancias eventuaes.

Diametros. Não cogitaremos dos diametros da grande bacia, com que se pretende determinar, indirectamente, o valor dos do E. S., nem do losango de Michaelis, cujo estudo. melhor se enquadra na pelvimetria.

\section{Antero-posteriores.}

\section{Estreito Superior}

C. A. (conjugado anatomico) ou P Supra P. (promonto-supra-pubico). Do promontorio á borda superior da symphyse pubica $=11$ ets.

C. V. (conjugado verdadeiro) ou P. P M. (promonto-pubico minimo), diametro util de Pinard, ou C. Obs. (conjugado obstétrico). Do promontorio ao ponto retro-pubico, isto é, ao ponto de Crouzat, que fica $5 \mathrm{mms}$. abaixo da borda superior da symphyse pubica $=10 \mathrm{1} / \mathrm{\%}$ cts. (francêses); ois allemães, em geral, dão-lhe 11 cts.

C. D. (conjugado diagonal) C. Obl. (conjugado obliquo); P Sob P (promonto-sob-pubico). Do promontorio ao ligamento arqueado. Não é propriamente diametro cujo tamanho seja mistér conhecido; é, porém, um auxiliar, graças ao qual se determinain as dimensões do C. V. Mede 12 cts. Delle subtrahe-se, em média, $1 \frac{1}{2}$ ct. para avaliar-se o C. V.

Obliquos. São aquelles que maior praticabilidade proporcionam á cabeça do féto.

Vão da eminencia ileo-pectinea á symphyse sacro-iliaca do lado opposto. Distinguem-se em esquerdo e direito. Para os francêses, esquerdo é o que parte da eminencia ileo-pectinea esquerda; direito, o que tem como ponto de inicio a direita. Para os allemãés, 'é o inverso: o ponto de partida é a symphyse sacro-iliaca.

Afím de dissipar a confusão que produz tal desacôrdo, Bar suggeriu, em 1902, no Congreaso de Roma, que se denominasse de $1 .^{\circ}$ obliquo ao esquerdo, e de $2 .^{\circ}$ obliquo ao direito, baseando-se no criterio clinico da maior frequencia de posições no diametro obliquo esquerdo (O. E. A. - $60 \%$; O. D. P. - $30 \%$ ). O diametro obliquo esquerdo mediria $127 \mathrm{mms}$; explicando-se dest'arte o maior numero de apresentações em O. E. A. e O. D. P.

Auvard emprega as designações de cocal erectal para os diametros obliquos esquerdo e direito, porque indicam, respectivamente, a direcção do ceco e a do recto.

\section{Transversos}

Tr. Cl. Transverso medio, util ou clinico, comprehendido dentro de pontos equidistantes do promontorio e symphyse pubica. Mede 12,8 cts. Casos ha em que é utilizado pela cabeça fetal.

Tr. max. Transverso maximo, bis-iliaco ou anatomico. De um a outro ponto mais distante da linha innominada. Mede $13 \mathrm{1} / 2 \mathrm{cts}$. Impraticavel, por se achar muito proximo do promontorio.

Tr. Kehrer. Transverso de Kehrer ou inter-ileo-pectineo. Vae de uma a outra eminencia ileo-pectinea. E' igual a 12 cts.

Inaproveitavel, porque está muito junto á symphyise púbica.

o conhecimento da dimensão do transverso anatomico e da do de 
Kehrer é precioso, quando se tenha de formular o prognostico do parto em bacias viciadas.

S. Cot. Sacro-cotyloide de Velpeau. Quasi perpendicular aos obliquos. Corre do promontorio ao ponto que corresponde ao vertice do cótylo; mede 9 cts. $\mathrm{E}^{\prime}$ interessante, mórmente, na mecanica do parto, em bacias coxalgicas.

Pr. Pect. Promonto-pectineo ou microcorda. Do angulo sacro-vertsbral á saliencia ileo-pectinea. Vale 9 a $9, \overline{0}$. Calculam-se-lhe as dimensões pela pelvigraphia. Informa da symetria da bacia e da capacidade das respectivas metades lateraes.

Arco anterior. Fochier -denominou arco anterior ao semi-anel pelvico do E. S., que fica adeante do diametro transverso medio. Tem um raio de 6 a 6 1/, cts.

A cabeça fetal sempre se põe em relação com o arco anterior.

Pelas noções que póde ministrar, elle denuncia o valor dos diametros transversos, cuja apreciação é excellente subsidio ao estabelecer-se o prognostico do parto.

E', portänto, de summo interesse determinar a relação do arco anterior com o diametro transverso mediano.

Graçaí a essa relação e ao C. V., póde-se classificar as bacias, sob o ponto de vista clinico, do modo seguinte:

Arco anterior normal, promonto-pubico minimo conservado = Bacia normal.

Arco anterior, fraca curvatura, C. V. reduzido $=$ Bacia achatada.

Arco anterior, fraca curvatura, C. V. superior a 9 cts. $=$ Bacia geralmente estreitada.

Arco anterior, fraca curvatura, $\mathrm{C}$. $\mathrm{V}$ abaixo de 9 cts. = bacia achatada e geralmente estreitada.

Arco anterior asymetrico, promontorio fóra do plano sagital do corpo = Bacia asymetrica.

Durante o parto, o arco anterior submette a cabeça a uma posição e attitude que lhe vem trazer a contacto a circumferencia cephalica que melhor adaptada se lhe mostra á forma; inversamente, a posição e attitude da cabeça fetal autorizam diagnosticar a conformação do arco anterior. D'ahi:

Posição transversa, attitude indifferente ou intermediaria, = arco anterior de fraca curvatura, ' bacia achatada.

Posição obliqua, flexão simples, arco anterior de curvatura normal, . bacia normal.

Posição transversa, flexão forçada, = arco anterior de forte curvàtura, $\because$ bacia achatada e geralmente estreitada.

Posição obliqua, flexão forçada $=$ arco anterior de forte curvatura, bacia geralmente estreitada.

Posição antero-posterior, = anco anterior de forte curvatura, bacia transversalmente estreitada. Muitas vezes, os phenomenos plasticos, calcados no craneo do recemnascido, vêm notificar o parteiro da forma do arco anterior.

\section{Escavação}

Antero posteriores.

m. S. m. P. (meio sacro meio pubico). Do meio da face-anterior do sacro ao meio da face posterior da symphyse pubica. Vale 12 cts.

S. Sob. P. Tem $111 / 2$ a 12 cts.

Transverso.

Bi. Sc. (Bi-sciatico). Entre as espinhas sciaticas $=10,8$. 
Obliquo (centro do buraco ischio-pubico á borda do pequeno ligamento sacro-sciatico), $=11$ cts.

Os diametros da escavação não medem indifferentemente 12 cts. como affirmam alguns autores. Reduzem-se, pela interposição de tecidos molles, de 3 a $5 \mathrm{mms}$. no algarismo colhido em bacia secca.

\section{Estreito inferior.}

Antero-posterior. C. Sob. P. (coci-sob-púbico) ou conjugata exitus. Da extremidade do cocix ao sob-pube. Mede 8,5 a 9 ets., podendo alcançar, pela retropulsão do cocix, até 11 cts.

Transverso. B. Isch. (Bi-Ischiatico). Da parte media da face interna de um a outro ischio; soffre reducção pela presença dos obturadores e da gordura ischio-rectal. Mede 11 cts.

Obliquo. Do meio do pequeno ligamento sacro-sciatico ao meio do ramo ischio-pubico. Tem 11 cts.

$\mathrm{Ha}$ ainda a considerar o esteno corda anterior de Von Ritgen, (sciatico-sob-pubico), que vae da espinha sciatica á borda inferior da symphyse. E' de muito apreço no que respeita á symetria do E. I.

\section{Diametros da bacia (resumo)}

Estreito superior

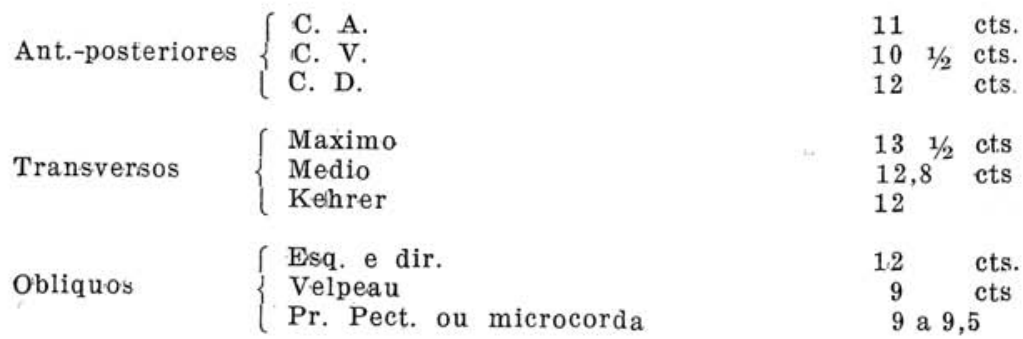

Escavação

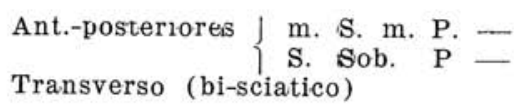

Obliquos

Estreito Inferior
Transverso (B. Isch.)

Obliquos

Antero-posterior

C. Sob. P.

$$
\begin{array}{lllll}
11 & 1 / 2 & \text { a } & 12 & \text { cts. } \\
10,8 & & \text { cts. }
\end{array}
$$

11 cts.

Inclinação. Inclinação pelvica é o angulo formado pelo C. V., ou plano do E. S., com a horizontal.

Em attitude erecta, a inclinação do E. S., é de $60 .^{\circ}$, e a do E. I. de $10 .^{\circ}$, os angulos respectivamente abertos para baixo e para cima.

A inclinação da bacia varia com a maior ou menor teneão dos ligamentos ileo-femoraes. 
A' vista daś diversas oscillações, consecutivas ás differentes pos.ções assumida pelo corpo, e attendendo a que a rotação do sacro em torno a seu eixo transverso (centro da $3 .^{\mathrm{a}}$ sacra), além de ser maís ou menos immovel, influencfa muito o phenomeno, Meyer lembrou o conjugado superior da symphyse; mede $300^{\circ}$, e suas variações não ultrapassam $50^{\circ}$.

Praticamente, sabe-se que a inclinação pelvica é normal quando a secção do acetábulo é francamente vertical, ou quando a espinha iliaca antero-posterior e o pube estão em um plano vertical.

Deve-se ainda indagar do angulo creado pela face posterior da symphyse com o C. V Mede $90^{\circ}-100^{\circ}$, porém, varia consoante á fórma, altura e inclinação symphysiaria.

Consignemos, por fim, o angulo sacro-vertebral, de abertura pos̀terior igual a $120^{\circ}$ e que, no decubito dorsal, indica de quanto é a lordose lombar.

Planos. Para referir as relaçóes entre o féto e os diversos pontos do canal pelvi-genital, conceberam-se planos ou circumferencias tocando zonas determinadas. Serão tantos quantos os pontos por onde tiverem de parsar.

Entre outros, merecem especial attenção aquelles descriptos por Hodge, de Philadelphia, em 18:65, e segundo os quaes fica demonstrado que o féto, em seu exito do organismo materno, transpõe qualtro planos, entre si parallelos.

1. Passa pelo estreito superior.

$2 .^{\circ}$ De todos o mais importante. Interessa atrás, o meio da $2 .^{\mathrm{a}}$ vertebra sacra; adeante, a borda inferior da symphyse pubica. Chamado, em geral, plano de Hodge, é circular e o mais espaçoso. Passa abaixo dos psoas-iliacos e acima dos pyramidaes, compromettendo apenas as fibras elevadas dos obturadores internos.

$3 .^{\circ}$ Córta as espinhas sciaticas; adeante os ischios e, atrás, a $5 .^{a}$ vertebra sacra.

$4 .^{\circ}$ Passa pela sob-pube e extremidade cocigea.

Movimentos da bacia. A embebição gravidica amplia, sobremodo, o jogo das articulações pelvicas e a distensibilidade dos ligamentos sacro-sciaticos.

A clinica aproveita-se desse estado particular para augmentar, conforme as circumstancias, este ou aquelle diametro do E. S. ou do E. I.

o parteiro recorre a tres posições obstetricas especiaes, estabelecidas nos movimentos do sacro.

1.a Posicão đe Crouzat-Walcher. Accresce o C. V. de 8 a 15 mms. (Dührssen, Fothergill, Küstner), graçass á contra-nutação sacra. A parturiente fica com os membros inferiores em hyperextensão, pendentes, repousando a região sacra sobre a borda de um plano. Favorece a insinuação. Esta posição é mais louvada pelos allemães que pelos francêses; estes, taes como Bar, Varnier, Bonnaire, Búé, não a julgam capaz de engrandecer o C. V além de $31 / 2 \mathrm{mms}$.

$\mathrm{O}$ crescimento dimensório se verifica por exagerar-se a lordose lombar e, particularmente, abaixar-se o arco anterior traccionado pelos membros inferiores. Este abaixamento é consecutivo ao basculo dos iliacos sobre o sacro immobilizado.

Sua melhor indicação observa-se nas applicações de fórcepe, maximé em cabeça ultima, quando esta occupar a escavação.

2. Posição de Laborie-Duncan. Amplifica o B. Isch. de 16 a 18 mms. Aqui, age a nutação. A mulher conserva-se na posição de 
talha, com os seus membros inferiores em hyperflexão, as coxą em flexão sobre a bacia, e as pernas sobre as coxas.

E' a posição chamada joelhos em frente ás espaduas.

A mencionada nutação sacra promove a rotação para dentro daç asas iliacas, e a rotação para fóra das tuberosidades ischiaticas.

Sua indicação electiva reside:

1. Nas apresentações de fronte, quando a cabeça é deficientemente reductivel na parte inferior da escavação, e quando ella procura transpor o E. S., segundo o diametro bi-ischiatico.

$21^{\circ}$ Nas bacias cyphoticas, quer o parto seja espontaneo, quer intervencionista.

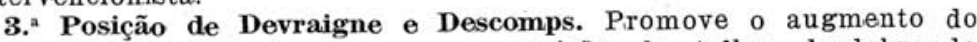
B. Isch. A parturiente conserva a posição de talha, desdoibrando as pernas; a attitude é analoga á do féto em apresentação pelvica, modo de nadegas. Obtem-se, dess'arte, a tensão dos musculos seguintes, adductores e posteriores da coxa, que se inserem na perna: recto interno, semi-membranoso, semi-tendinoso e biceps. Em contracção, elles exceutam um maximo de tracção sobre cada ramo ischio-pubico, onde ficam as suas inserções superiores. Uma ligeira abducção das coxas, de mais ou menos $45^{\circ}$, põe em relaxamento os grandes gluteos.

As parturientes, instinctivamente, no decurso do parto, variam ? attitude do corpo, conforme o periodo do trabalho.

Durante a dilatação, isto é, quando a cabeça fetal ainda apoia sobre o estreito superior, ella se mantem de pé, sentada ou em decubito dorsal, em extensão que não fatigue (posição de CrouzatWalcher).

$\mathrm{Na}$ expulsão, tendo a cabeça percorrido a escavação e chegando á vagina, a parturiente dobra e eleva as pernas, inclina para deante o tronco e provoca, por esse mecanismo, modificações pelvicas que facilitem a descida do féto (posição de Laborie-Duncan).

O facto é tanto mais significativo por saber-se que a propulsão do corpo produz o afastamento da ponta do sacro e, como conøequencia, o augmento do estreito inferior.

Factor ethnico. A frequencia de gestantes, originarias da raça africana, manda que se considere a influencia do factor ethnico sobre o feitio da bacia, e, ergo, sobre a marcha do trabalho.

Em 1904, Riggs, de-Baltimore, chegou a conclusões que podem ser subscriptas pela Maternidade de S. Paulo.

As anguistias da bacia ossea são mais encontradiças em pretas que em brancas, na proporção de $34 \%$, para aquellas, e $9 \%$ para estas. No entretanto, pratica-se maior numero de intervenções nas brancas, porque, nas pretas, a cabeça fetal é menos volumosa e gosa de maior compressibilidade.

A bacia de negra é mais estreita e parece mais profunda.

0 filho de branca mede mais $1 \frac{1}{2}$ ct. e pesa mais $200 \mathrm{grs}$. que o da preta.

A frequencia das apresentações cephalicas é relativamente maior - nas negras.

Ao juizo de Tarnier, o diametrò obliquo do estreito superior, na raça negra e mongolica, differe do transverso apenas de algunis millimetros, ao passo que, na aryana, a divergencia é de centimetro e meio.

Lazary, no Registro da Maternidade do Rio, (n. 14-1916), esmiuçando os eneinamentos de 939 casos obstetricos, verificou que a pelvimetria de bacias anómalas, dava, segundo a côr das mulheres. as seguintes percentagens: 


$\begin{array}{ccc}\text { Brancas: } & \text { Pardas: } & \text { Pretas: } \\ 15,245 \% ; & 18,012 \% ; & 23,032 \% .\end{array}$

Esses algarismos corroboram o nosso asserto.

Para concluir, assignalemos a documentação numerica com que o Dr. Clovis Corrêa da Costa, em seu trabalho - Rupturas do perineo - Influencia ethnica (Archivos Brasileiros de Medicina. Dezembro, 1917), corrobora a observação quotidiana de que o perineo de preta é mais roturavel que o da parda, e o desta mais que o de branca. Os algarismos referem-se á Materninade do Rio.

$\begin{array}{lrcc}\text { Pretas: } & & \text { Pardas: } & \text { Brancas: } \\ \text { Iparas: } & 54,3 \% ; & 40,8 \% & 34 \% \\ \text { Multiparas: } & 9,5 \% ; & 6,97 \% & 461 \%\end{array}$

\section{Boletim do \\ Gremio dos Internos dos Hospitaes}

Em 10 de abril p. p., foi fundado o "Gremio dos Internos dos Hospitaes", da Faculdade de Medicina e Cirurgia de S. Paulo, tendo-se a 1.a sessão realisado na séde do Centro Academico "Oswaldo Cruz", cedida pelo seu presidente.

Lançadas as bases da novel a'gremiação, foi, desde logo, nomea. da uma commissão encarregada de elaborar os Estatutos sociaes, e comporsta pelos Srs. Souza Campos, Toledo Mello, Flaminio Favero, Romeu da Silveira e Benjamin Reis.

A segunda sessão "do "Gremio dos Internos" teve lugar no dia 17 de abril, na séde da Sociedade de Medicina e Cirurgia, gentilmente cedida pela directoria.

Por essa accasião, foram discutidos os Estatutos elaborados e eleita a $1 .^{\text {a }}$ directoria que deve dirigir os destinos do Gremio no periodo 1918 1919 , ficendo assim comstituida: presidente, doutorando José de Toledo Mello; vice-presidente, quintannista Ernesto Moreira; $1 .^{\circ}$ secretario, doutorando Flaminio Favero; $2 .^{\circ}$ secretario, quintannista Joaquim Queiroz e thesoureiro, doutorando Simeão dos Santosı Bomfim.

INa $3 .^{*}$ sessão preparatoria, realisada no dia 27 de abril, na séde da Sociedade de Medicina, foram approvados os Eistatutos sociaes, tendo sido, então, perfieitamente estabelecidos os fins da associação, e que se resumem. no seguinte:

"Celelbrar sessões em que sejam estudados e discutidos assumptos que dizem respeito ás disciplinas professadas nos cursos da Faculdade de Medicina e Cirurgia de S. Paulo; publicar, na "Revista de "Medicina", os trabalhos dos socios; deffender os interesses dos seus associados, dentro da sua alçada; promover conferencias 